\title{
Evaluation of the Early Functional Outcome Following Arthroscopic Partial Meniscectomy for Meniscal Tears
}

\author{
Francis 0. Okanu ${ }^{1,2}$, Kelechukwu M. Onuoha1,2, Shopekhai Itakpe ${ }^{1,2}$ \\ ${ }^{1}$ Department of Surgery, National Orthopedic Hospital, Igbobi Yaba, Lagos State, Nigeria \\ ${ }^{2}$ Department of Orthopedic Surgery, Babcock University Teaching Hospital, Ilishan Remo, Ogun State, Nigeria \\ Email: mckelng@yahoo.com
}

How to cite this paper: Okanu, F.O. Onuoha, K.M. and Itakpe, S. (2020) Evaluation of the Early Functional Outcome Following Arthroscopic Partial Meniscectomy for Meniscal Tears. Journal of Biosciences and Medicines, 8, 32-42.

https://doi.org/10.4236/jbm.2020.812004

Received: October 23, 2020

Accepted: December 11, 2020

Published: December 14, 2020

Copyright $\odot 2020$ by author(s) and Scientific Research Publishing Inc. This work is licensed under the Creative Commons Attribution International License (CC BY 4.0).

http://creativecommons.org/licenses/by/4.0/

\begin{abstract}
Meniscal tears are among the common knee pathologies which affect activities of daily living if not managed properly. Arthroscopic knee surgery is an evolving procedure in our environment and patient satisfaction with this procedure needs to be evaluated. This was a prospective study carried out between June 2017 and May 2018 with the aim to determine the effectiveness of Arthroscopic Partial Meniscectomy in the management of meniscal tears in our environment. The Western Ontario Meniscal Evaluation Tool (WOMET) Knee Score was used to assess patient satisfaction with this procedure. A total of thirty-one patients were recruited into the study consisting of eighteen males and thirteen females with an average age of thirteen years (17 - 48 years) who underwent arthroscopic partial meniscectomy. Preoperative and postoperative knee scores at 6 weeks and 12 weeks were compared using the Western Ontario Meniscal Evaluation Tool. At the end of the study period, data collated were analyzed using the specified tools. In terms of clinical outcomes, arthroscopic partial meniscectomy showed statistically significant improvement of symptoms as evidenced by a mean knee score of 75.6 (SD 9.3) at 6 weeks, 87.7 (SD 4.7) at 12 weeks compared with a preoperative knee score of 46.7. Medial meniscal tears were more common than lateral meniscal tears in all age groups and both sexes recruited into the study. The most common type of meniscal tear seen was the longitudinal type of tear while complex tear is the least type of tear seen. The surgery (arthroscopic partial meniscectomy) is an evolving area of sports medicine that requires sub-specialization, however solves the problem of increased mobility following open surgery. This study will tend to add to existing knowledge as patients who are mostly sports inclined can return to play very early with better outcome scores as regards pain and function.
\end{abstract}




\section{Keywords}

Meniscal Tear, Arthroscopy, Partial Meniscectomy, Western Ontario Meniscal Evaluation Tool (WOMET) Knee Score

\section{Introduction}

The use of arthroscopy in the diagnosis and treatment of knee pathologies has evolved all over the world and its use is currently on the rise in Sub-Saharan Africa. A few studies have been done to assess the effectiveness of these procedures in our environment [1] [2]. A torn meniscus is one of the most common reasons why patients undergo arthroscopic surgery [3]. Meniscal tear is the most common injury of the knee, with an incidence of 61 per 100,000 populations per year in the United Kingdom [4]. Arthroscopic partial meniscectomy is one of the treatment modalities for meniscal tears which may result from trauma, sport injuries and degenerative knee conditions. Although arthroscopic partial meniscectomy has been noted to increase load transmission across the knee joint predisposing it to osteoarthritis in the long run [5], some authors have argued that patients with meniscal injury in some selected cases will benefit from non-operative management [6]. It is therefore important to evaluate the benefits of arthroscopic partial meniscectomy. Diagnosis of meniscal tear was made clinically and confirmed during knee arthroscopy (Figure 1).

Factors such as age, sex and weight have been documented to influence the postoperative outcome following arthroscopic partial meniscectomy [7]. The incidence of meniscal tears is high in knees with ruptures of the anterior cruciate ligament [8].

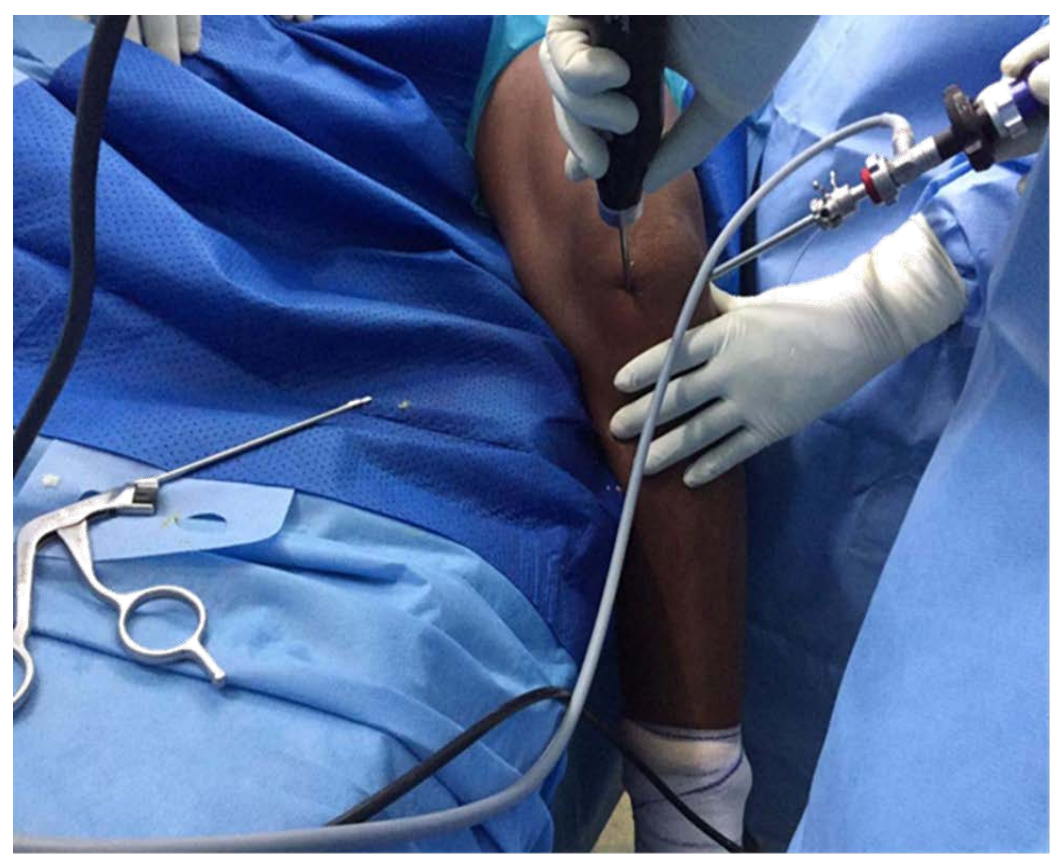

Figure 1. Ongoing knee arthroscopy surgery. 


\section{Materials and Method}

\subsection{Study Design}

The study was a prospective interventional study aimed at evaluating the functional outcome of arthroscopic partial meniscectomy in patients with unilateral meniscal tears. The preoperative and postoperative knee score at 6 weeks and 12 weeks were assessed using the WOMET knee score.

\subsection{Sample Size}

The sample size of 31 was calculated using the formulae developed by Cochran et al. [9] Formulae $n_{0}=\frac{Z^{2} p q}{d^{2}}$.

\subsubsection{Setting}

This study was carried out at the Arthroscopy unit of the National Orthopaedic Hospital, Igbobi Lagos.

\subsubsection{Data Source}

All patients recruited for the study had an initial counseling, after which a patient information sheet was provided which introduces the researcher and provides basic information concerning the research. Informed consent was obtained before recruitment into the study.

\subsection{Statistical Methods}

The data obtained was analyzed using statistical software (SPSS version 21.0). Continuous variables were summarized by mean and standard deviation and comparison made by using student $\mathrm{t}$-test.

Categorical variables were summarized by frequency tables and comparisons were made using the chi square or Fischer's exact test. The level of significance was set at $5 \% . \mathrm{P} \leq 0.05$.

\subsection{Inclusion Criteria}

- Patients with symptomatic meniscal tears affecting only one knee.

- Patients with arthroscopically diagnosed meniscal tears not amenable to repair.

- Patients with unilateral osteoarthritis of the knee (Kellgren and Lawrence stage 1) with coexisting meniscal tear.

\subsection{Exclusion Criteria}

- Patients with meniscal tears affecting both knees.

- Patients with associated ligamentous injuries.

- Patients with coexisting femoral and tibia fractures.

- Patients with deformities of the lower limbs.

- Patients with active knee infections.

- Patients with grade II and above (Kellgren and Lawrence) Osteoarthritis of 
the knee with degenerative tears.

\subsection{Ethical Approval}

Ethical clearance was obtained from the Ethical and Medical Research Committee of the hospital.

\subsection{Methods}

First contact was made with the patient in the outpatient department and the following preoperative steps were taken. Biodemographic data, duration of symptoms, mechanism of injury, history of pain, history of locking and giving way and swelling was noted at the time of presentation. The presence of joint line tenderness, a positive Mcmurray's test, Apley's grind test and range of motion of the knee were documented.

All patients had radiographs of the affected knee and magnetic resonance imaging. Informed consent was obtained with details of the treatment and the possible complications were explained to the patient. Preoperative knee scores of the affected knee were documented using the WOMET knee score.

\subsection{Operative Procedure}

- The procedure began with an examination of the knee under anaesthesia. The anterolateral viewing portal was placed approximately $1 \mathrm{~cm}$ below the inferior pole of the patella and roughly $1 \mathrm{~cm}$ lateral to the lateral border of the patellar tendon.

- The portal was made as an approximately $1 \mathrm{~cm}$ vertical incision with the blade of the scalpel.

- Turned upward and away from the meniscus. The anteromedial portal was made under direct visualization with the arthroscope in the anterolateral portal and portal position was localized.

A thorough diagnostic arthroscopy was completed to identify all areas of pathology. Following diagnostic arthroscopy and identification of a tear, the characteristics of the tear: type, location, size, stability and associated pathologies in the knee were noted.

The tear was carefully evaluated to determine if the tear was repairable and if the meniscal tissue had a high index of healing. Partial meniscectomy was undertaken after determination that meniscal repair was not possible.

Meniscal baskets and arthroscopic shavers were used to resect all mobile fragments while preserving as much functional meniscus as possible. After partial meniscectomy was complete, the remaining meniscus was reassessed and probed to confirm that the remaining meniscus was stable. Nylon 2/0 was used to close the wound in a single layer. A Karl Storz Arthroscopy tower was the machine used for the arthroscopic surgery as seen in Figure 2.

\section{Results}

A total of 37 patients were recruited into this study out of this number, 3 patients 


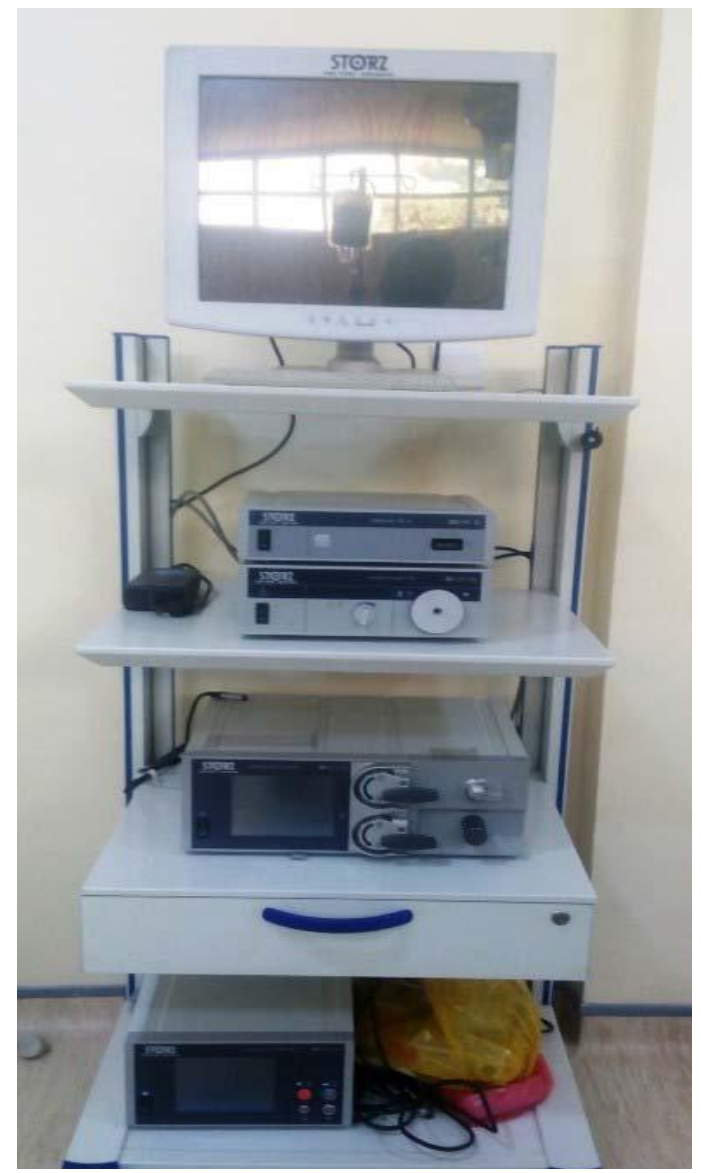

Figure 2. Karl Storz Arthroscopy tower.

were lost to follow up. 3 other patients failed to keep regular clinic appointments. On account of these 31 patients with meniscal tears completed the study.

\subsection{Age Distribution}

The age range of the study population was 17 - 48 years with a mean age presentation of $=31.45 \pm 9.53 .50 \%$ of patients were in the $4^{\text {th }}$ decade while those under 20 years of age contributed to only 16\%, as seen in Figure 3 .

\subsection{Sex Distribution}

18 of the patients were males (58\%) were males while 13 of the patients were (42\%) female with a male to female ratio of 1.4:1, as seen in Table 1.

\subsection{Aetiology of Meniscal Injuries among the Subjects}

Although none of the patients were professional athletes, 14 (45.2\%) patients sustained meniscal injuries during sporting activities. $5(16.1 \%)$ sustained injury during a fall, 4 (12.9\%) from motorcycle accidents, 2 (6.1\%) were pedestrian accidents while $6(19.4 \%)$ sustained injury from no identifiable cause, as seen in Figure 4. 


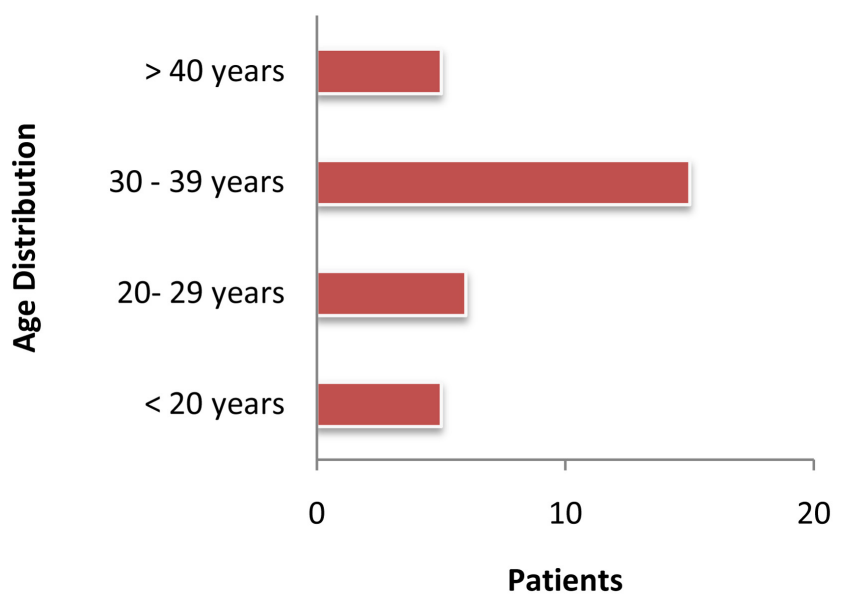

age distribution

Figure 3. Distribution of age.

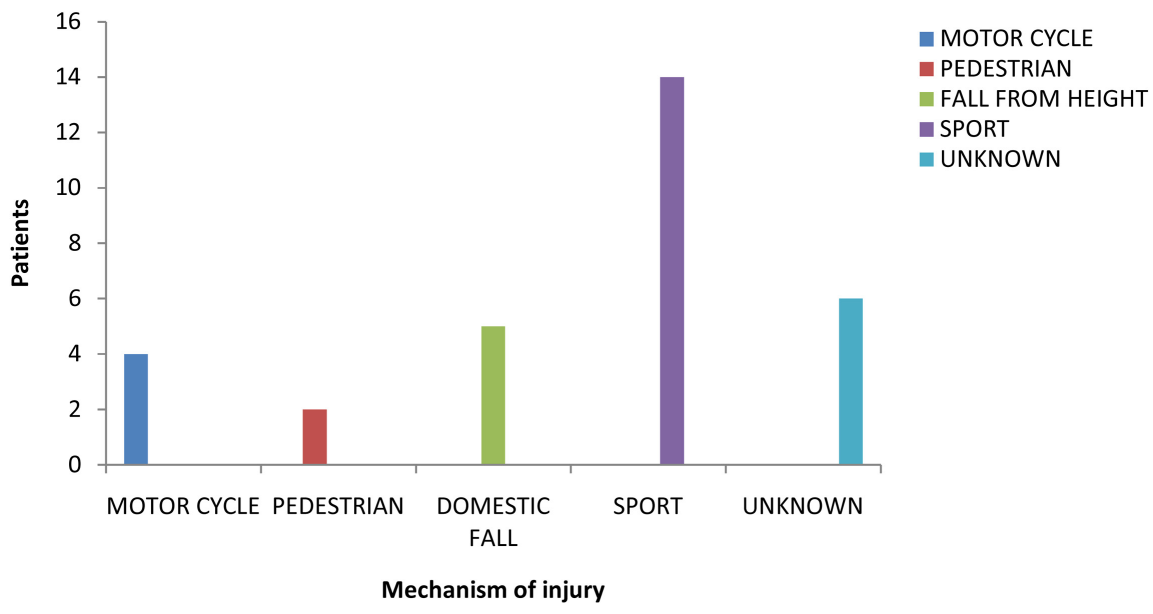

Figure 4. Aetiology of meniscal injuries.

Table 1. Sex distribution.

\begin{tabular}{ccc}
\hline Sex & Frequency & Percentage \\
\hline Male & 18 & 58 \\
Female & 13 & 42 \\
Total & 31 & 100 \\
\hline
\end{tabular}

\subsection{Distribution of Patients by Knee Involvement}

Of the 33 unilateral knees with meniscal tears 19 affected the right knee while 12 affected the Left knee, accounting for $61.3 \%$ and $38.7 \%$ respectively, as seen in Table 2.

\subsection{Pattern of Meniscal Tears}

The types of meniscal tears in our study ranged from Longitudinal tears seen in 16 patients (52\%), Radial tears 9 patients (29\%), Flap tears 2 patients (6\%), Complex tears 4 patients (13\%), as seen in Figure 5. 
Table 2. Distribution of patients by knee involvement.

\begin{tabular}{ccc}
\hline Knee & Frequency & Percentage \\
\hline Right & 19 & $61.3 \%$ \\
Left & 12 & $38.7 \%$ \\
Total & 31 & $100 \%$ \\
\hline
\end{tabular}

\title{
PATTERN OF MENISCAL TEARS
}

\author{
- LONGITUDINAL $\quad$ RADIAL $\square$ FLAP $\quad$ COMPLEX
}

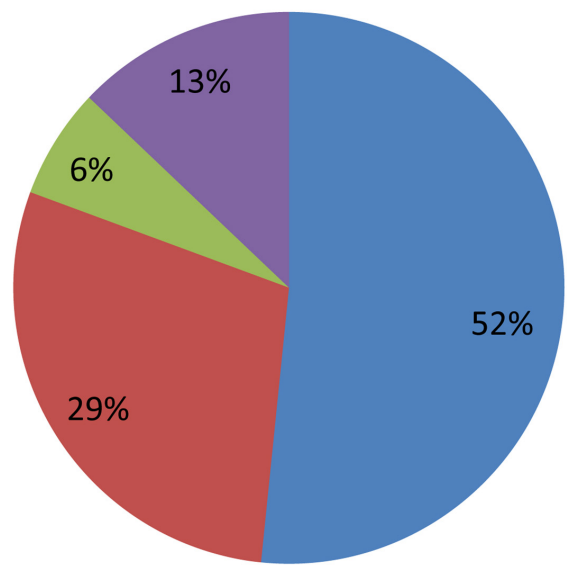

Figure 5. Pattern of meniscal tears.

\subsection{Location of Tears in Patients Who Had APM}

Of the 31 patients had APM with $90 \%$ of tears located in the white-white zone and $10 \%$ of tears located in the red-white zone. None of the tears in the red-red zone had APM, as seen in Figure 6.

\subsection{Baseline and Early Functional Outcome of Subjects Who Had Arthroscopic Partial Meniscectomy}

Prior to surgery the WOMET knee score was 46.7 with an S.D of 16.3. At 6 weeks post op the knee scores were 75.6 with an S.D of 9.3. At 12 weeks post-operative knee scores were 87.7 with an S.D of 4.7 as seen in Table 3 .

\subsubsection{Relationship between Improvement in Early Function Outcomes at 12 Weeks and Demographic Variables}

There was no significant relationship in the difference between preoperative knee score at 12 weeks knee score and the various age groups with $\mathrm{p}$ value $>$ 0.05 . The relationship between knee score difference and sex had a $p$ value $>0.05$ which is not statistically significant as seen in Table 4 .

\subsubsection{Relationship between Meniscal Tears and Knee Score}

Patients with lateral meniscal tears had better mean Knee scores when compared with the medial meniscal tears both in preoperative, postoperative at $6^{\text {th }}$ week 


\section{LOCATION OF TEARS}

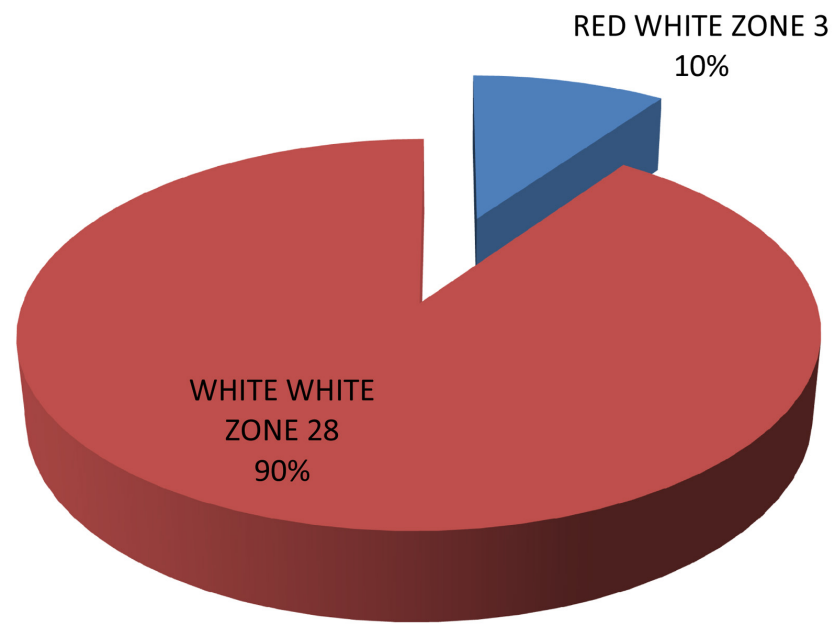

Figure 6. Location of tears in patients who had APM.

Table 3. Baseline and early functional outcome of subjects who had arthroscopic partial meniscectomy.

\begin{tabular}{ccccc}
\hline VARIABLE & MEAN & S.D & $\begin{array}{c}\text { MINIMUM } \\
\text { VALUE }\end{array}$ & $\begin{array}{c}\text { MAXIMUM } \\
\text { VALUE }\end{array}$ \\
\hline $\begin{array}{c}\text { PRE-OPERATIVE KNEE } \\
\text { SCORE }\end{array}$ & 46.7 & 16.3 & 22.7 & 74.6 \\
$\begin{array}{c}\text { POST-OPERATIVE KNEE } \\
\text { SCORE AT 6 WEEKS }\end{array}$ & 75.6 & 9.3 & 60.1 & 92.9 \\
$\begin{array}{c}\text { POST-OPERATIVE KNEE } \\
\text { SCORE AT 12 WEEKS }\end{array}$ & 87.7 & 4.7 & 79.5 & 95.6 \\
\hline
\end{tabular}

Table 4. Relationship between improvement in early function outcomes at 12 weeks and demographic variables.

\begin{tabular}{ccccc}
\hline Age group & $\begin{array}{c}\text { Knee score difference. } \\
\text { Mean }\end{array}$ & SD & F-statistics & P-Value \\
\hline$<20$ & 44.9 & 13.3 & 0.271 & 0.846 \\
$20-29$ & 42.3 & 12.2 & & \\
$30-39$ & 39.3 & 12.5 & & \\
$\geq 40$ & 40.5 & 13.1 & & 0.634 \\
Sex & & & -0.481 & \\
Male & 39.9 & 14.3 & & \\
Female & 42.1 & 9.8 & & \\
\hline
\end{tabular}

and postoperative at $12^{\text {th }}$ week with $\mathrm{p}$ value $<0.05$ as seen in Table 5 .

\section{Discussion}

Meniscal lesions are among the commonest knee injuries and arthroscopy is the 
Table 5. Relationship between meniscal tears and knee score.

\begin{tabular}{cccccc}
\hline Knee Score & & Mean (\%) & S.D & t-statistics & p-value \\
\hline \multirow{2}{*}{ Preoperative } & Medial & 39.2 & 14.9 & -3.739 & 0.001 \\
& Lateral & 59.3 & 11.3 & & \\
Postoperative & Medial & 70.8 & 7.8 & -4.665 & 0.015 \\
(6 weeks) & Lateral & 84.1 & 6.0 & & \\
Postoperative & Medial & 86.7 & 4.3 & -3.637 & 0.001 \\
At 12 weeks & Lateral & 91.4 & 3.4 & & \\
Difference At & Medial & 46.5 & 11.1 & -3.543 & 0.001 \\
12 weeks & Lateral & 32.1 & 9.0 & & \\
\hline
\end{tabular}

best method of diagnosing and managing these lesions. The meniscus is recognized for its role in normal knee biomechanics and efforts are made to preserve the meniscus to prevent degenerative osteoarthritis, however not all meniscal tears can be repaired [1].

Eighteen (58\%) patients were males and $13(42 \%)$ patients were females with a male to female ratio of 1.4:1. This male preponderance is similar to findings by Maffulli et al. [5] whose study had a male to female ratio of 2.5:1, and that by Nilton et al. [10] who had a male to female ratio of 5.5:1. Sports injuries were the most common cause of meniscal tears in this study accounting for 14 (45.2\%) cases. Eleftherious et al. [11] reported sport injuries accounting for a third of the cases Seen in their study. 6 (19.4\%) patients sustained meniscal tears from non-traumatic causes but a Study by Englund et al. [12] gave a prevalence of non-traumatic meniscal tears of $31 \%$.

Duration of symptoms was an average of 23 months with earliest presentation at 4 months while 60 months was the largest duration before presentation. This may be due to the lack of awareness of the treatment options available in the country by primary care givers and patients [13]. Pathania et al. [14] also reported that $42 \%$ of patients presented 2 years after onset of symptoms. Of the 31 cases of unilateral meniscal tears in this study, 19 (61\%) involved the right knee while $12(39 \%)$ affected the left knee, a ratio of $1.6: 1$. Thirteen $(68.4 \%)$ patients had medial meniscus tears in the right knee while $6(31.6 \%)$ patients had lateral meniscal tear in the right knee. In the left knee $8(66.6 \%)$ patients had tears in their medial menisci and 4 (33.4\%) patients had tears in their lateral menisci. This is probably due to the fact that the medial meniscus is less mobile than the lateral meniscus and more likely to be trapped between condyles when abnormal joint loading occurs.

APM was carried out on 31 patients with $90.5 \%$ of tears located in the white-white zone and $9.5 \%$ of tears extending into the red-white zone. This was contrary to the findings of Riley et al. [15] where the distribution of meniscal tears involved only the white-white zone of the meniscus in $10 \%$, extended into the red-white zone in $30 \%$, and extended into the peripheral third red-red zone in $60 \%$ of patients. Prior to surgery, the average knee score using WOMET score was 46.7. There was an increase in the mean knee score at 6 weeks and 12 weeks 
with knee scores of 75.6 and 87.7 respectively. These values were statistically significant $\mathrm{p}<0.05$, confirming patient satisfaction with the procedure and also suggested improvement of symptoms with time. Hong et al. [16] noted a significant increase in postoperative knee scores after APM at 3 months and 9 months compared with preoperative knee scores. Pathania et al. [14] also had good knee scores at 6 weeks and 9 months. The relationship between the age of our patients and the postoperative knee scores at 12 weeks was not significant with a $p>0.05$. This study also did not establish a statistically significant association between knee score and the sex of patient $\mathrm{p}>0.05$. Babalola OR et al. [17] reported that there was improvement in WOMET scores at 7 months post operatively with greater improvement with medial partial meniscectomy as opposed to partial lateral meniscectomy. This was however not statistically significant. This buttressed the fact that arthroscopic partial meniscectomy has a role in the management of meniscal tears in the short term.

\section{Limitation of Study}

- Some patients were lost to follow up due to poor clinic attendance.

- High cost of MRI scans was a financial burden for some patients which reduced the number of patients recruited into the study.

\section{Conclusion}

Arthroscopic partial meniscectomy for irreparable meniscal tears leads to improved knee function in the early post operative period as evidenced by improvement in WOMET knee scores and arthroscopic partial meniscectomy is a safe procedure with low complication rates.

\section{Acknowledgements}

We acknowledge the patients involved that gave their consent for publication.

\section{Conflicts of Interest}

The authors declare no conflicts of interest regarding the publication of this paper.

\section{References}

[1] Giwa, S. (2017) Arthroscopic Meniscectomy. Journal of the West African College of Surgeons, 7, 10-13.

[2] Olusunmade, O.I. and Salami, S.O. (2015) Arthroscopically Assisted Treatment of a Malunited Tibia Plateau Fracture; A Case Report. Annals of Nigerian Medicine, 9, 66-69. https://doi.org/10.4103/0331-3131.177957

[3] Briggs, K.K., Kocher, M.S., Rodkey, W.G. and Steadman, J.R. (2006) Reliability, Validity, and Responsiveness of the Lysholm Knee Score and Tegner Activity Scale for Patients with Meniscal Injury of the Knee. Journal of Bone and Joint Surgery-Series A, 88, 698-705. https://doi.org/10.2106/00004623-200604000-00003

[4] Amis, A.A. and McDermott, I.D. (2006) The Consequences of Meniscectomy. The 
Journal of Bone and Joint Surgery. British Volume, 88, 1549-1556. https://doi.org/10.1302/0301-620X.88B12.18140

[5] Campi, S., Denaro, V., Longo, U.G. and Maffulli, N. (2010) Meniscal Tears. Open Access Journal of Sports Medicine, 1, 45-54. https://doi.org/10.2147/OAJSM.S7753

[6] Seon, J.K., Song, E.K., Yim, J.H., et al. (2013) A Comparative Study of Meniscectomy and Nonoperative Treatment for Degenerative Horizontal Tears of the Medial Meniscus. American Journal of Sports Medicine, 41, 1565-1570. https://doi.org/10.1177/0363546513488518

[7] Lindeboom, R., Lucas, C., Snoeker, B.A.M. and Zwinderman, A.H. (2015) A Clinical Prediction Rule for Meniscal Tears in Primary Care: Development and Internal Validation Using a Multicentre Study. British Journal of General Practice, 65, 523-529. https://doi.org/10.3399/bjgp15X686089

[8] Aho, H., Järvelä, T., Järvinen, T.L.N. and Sihvonen, R. (2012) Validation of the Western Ontario Meniscal Evaluation Tool (WOMET) a Degenerative Meniscal Tear. The Journal of Bone and Joint Surgery. American Volume, 65, 8-15. https://doi.org/10.2106/JBJS.K.00804

[9] Cochran, W. (1977) Sampling Techniques. Third Edition, John Wiley \& Sons Inc., New York, 85-91.

[10] De Oliveira, N.H.C., de Souza Leão, M.G. and Orlando Júnior, N. (2015) Diagnosis of Knee Injuries: Comparison o the Physical Examination and Magnetic Resonance Imaging with the Findings from Arthroscopy. Revista Brasileira de Ortopedia (English Edition), 50, 712-719. https://doi.org/10.1016/j.rboe.2015.10.007

[11] Hanney, W.J. and Pabian, P. (2008) Functional Rehabilitation after Medial Meniscus Repair in a High School Football Quarterback: A Case Report. North American Journal of Sports Physical Therapy, 3, 161-169.

[12] Englund, M., Gale, D. and Guermazi, A. (2008) Incidental Findings on Knee MRI in Middle Aged and Elderly Persons. The New England Journal of Medicine, 359, 1108-1115. https://doi.org/10.1056/NEJMoa0800777

[13] Adekoya-cole, T.O., Akinmokun, O.I., Enweluzo, G.O. and Orakwe, D.E. (2011) Basic Arthroscopy-A Review Paper. Nigerian Quarterly Journal of Hospital Medicine, 21, 303-305.

[14] Arora, N.C., Kulshreshtha, V. and Pathania, V.P. (2001) Arthroscopic Evaluation and Management of Menisceal Injuries of the Knee. Medical Journal Armed Forces India, 57, 99-103. https://doi.org/10.1016/S0377-1237(01)80123-4

[15] Cordasco, F.A., Hatch, J., Petrigliano, F.A., Potter, H.G., Warner, K.K. and Williams, R.J. (2007) MRI Evaluation of Isolated Arthroscopic Partial Meniscectomy Patients at a Minimum Five-Year Follow-Up. HSS Journal, 3, 35-43. https://doi.org/10.1007/s11420-006-9031-2

[16] Cao, H., Cheng, X.H., Guo, X.P., Ke, Y., Zhang, Y. and Qian, W. (2012) Short-Term Clinical Outcomes of 42 Cases of Arthroscopic Meniscectomy for Discoid Lateral Meniscus Tears. Experimental and Therapeutic Medicine, 4, 807-810. https://doi.org/10.3892/etm.2012.686

[17] Alatishe, K., Babalola, O.R., Itakpe, S.E., Laiyemo, E.A., Okanu, F. and Madubueze, S.O. (2017) Arthroscopic Partial Meniscectomy-Short-Term Clinical Outcome in an Orthopaedic Center in Southwestern Nigeria. Journal of the West African College of Surgeons, 7, 1-8. https://doi.org/10.4103/ajmhs.ajmhs_2_17 